\title{
Preface \\ Message from the Guest Editors-In-Chief
}

\author{
Masatoshi OKutomi ${ }^{\dagger 1}$ and Hongbin ZhA ${ }^{\dagger 2}$
}

It is our pleasure to present this special issue on MIRU2009, the 12th Meeting on Image Recognition and Understanding held in Matsue, Japan, in July 2009. MIRU is one of the largest domestic conferences in Computer Vision and Pattern Recognition area of Japan and about two hundred fifty high-quality papers were presented in 2009.

This special issue contains five papers which is the extended version of the conference paper presented at MIRU2009. All submitted papers were subject to a strict peer-review by three reviewers and an associate editor assigned for each paper. We believe that the accepted papers, including an interesting invited paper entitled "Challenges in wide-area structure-from-motion", can provide certain contribution to the community.

Finally we would like to thank all those who made this special issue possible, including the authors for timely submitting and revising their papers, the associate editors and reviewers for dedicating a significant amount of time for maintaining the quality of this CVA special issue.

\section{Guest Editors in Chief:}

Masatoshi Okutomi Tokyo Institute of Technology

Hongbin Zha Peking University 